\title{
A Prospective Randomized Study Analyzing Preoperative Opioid Counseling in Pain Management After Carpal Tunnel Release Surgery.
}

Todd H. Alter

Rothman Institute at the Thomas Jefferson University

Asif M llyas

Rothman Institute, Thomas Jefferson University

Follow this and additional works at: https://jdc.jefferson.edu/rothman_institute

Part of the Orthopedics Commons

Let us know how access to this document benefits you

\section{Recommended Citation}

Alter, Todd H. and Ilyas, Asif M, "A Prospective Randomized Study Analyzing Preoperative Opioid Counseling in Pain Management After Carpal Tunnel Release Surgery." (2017). Rothman Institute Faculty Papers. Paper 94.

https://jdc.jefferson.edu/rothman_institute/94

This Article is brought to you for free and open access by the Jefferson Digital Commons. The Jefferson Digital Commons is a service of Thomas Jefferson University's Center for Teaching and Learning (CTL). The Commons is a showcase for Jefferson books and journals, peer-reviewed scholarly publications, unique historical collections from the University archives, and teaching tools. The Jefferson Digital Commons allows researchers and interested readers anywhere in the world to learn about and keep up to date with Jefferson scholarship. This article has been accepted for inclusion in Rothman Institute Faculty Papers by an authorized administrator of the Jefferson Digital Commons. For more information, please contact: JeffersonDigitalCommons@jefferson.edu. 


\section{As submitted to:}

\section{The Journal of Hand Surgery}

2017 Aug 16. pii: S0363-5023(17)30660-3. doi: 10.1016/j.jhsa.2017.07.003.

Todd H. Alter, Rothman Institute at the Thomas Jefferson University, Philadelphia, PA.

Asif Ilyas, Rothman Institute at the Thomas Jefferson University, Philadelphia, PA. Electronic address: asif.ilyas@rothmaninstitute.com.

\section{ABSTRACT}

Purpose: Prescription opioid misuse has become increasingly prevalent in the United States. Preoperative opioid counseling has been proposed to decrease opioid consumption after surgery. This study aimed to evaluate the effect of pre-operative opioid counseling on patients' pain experience and opioid consumption after carpal tunnel release (CTR) surgery.

Methods: A prospective comparison of consecutive patients scheduled to undergo CTR surgery was conducted. Patients were randomized to either receiving formal pre-operative opioid counseling or no counseling. All operations were performed with the same mini-open CTR surgical technique and the same number of opioids were prescribed post-operatively. Daily opioid pill consumption, pain levels, and any adverse reactions were recorded.

$\underline{\text { Results: }}$ : During the day of surgery and the first day following surgery, patients in the group with counseling reported significantly fewer prescribed opioid pills consumed, compared to patients in the group without counseling, while experiencing no significant difference in pain level experience. In addition, patients in the group with counseling reported a significantly lower 
number of total pain pills consumed over the course of the study than the group without counseling. No major adverse reactions were noted in either group.

Conclusions: Pre-operative opioid counseling was found to result in a significant decrease in overall opioid consumption post-operatively.

Level of Evidence: Therapeutic (Level II) 


\section{$\underline{\text { INTRODUCTION }}$}

Prescription opioid abuse has been a paramount issue in recent years as a result of its dramatic increase in prevalence in the United States and the negative effects associated with this increase to society at large. ${ }^{1-5}$ Between the years $1999-2010$, rates of opioid sales, deaths, and treatment admission have each more than tripled. ${ }^{1}$ In the same period of time, overdose deaths from pharmaceutical opioids represented the highest relative increase of overdose death rates among all drug types in the United States, with a nearly 4-fold increase. ${ }^{2}$ In total, there were 238 million narcotic analgesic prescriptions in the United States alone in 2011, with oxycodone and hydrocodone making up the majority. ${ }^{3}$ Many common elective and trauma-related orthopedic procedures have been identified as potentially resulting in prolonged opioid use, including CTR surgery, for which $76 \%$ of patients filled at least one prescription for opioid analgesics and $14 \%$ filled a prescription beyond 90 days postoperatively. ${ }^{4}$ Despite the already high rate of opioid use following common orthopedic surgeries, opioid prescribing for orthopedic surgeries continues to increase and inadvertent over-prescribing is a common phenomenon. ${ }^{5-6}$

Various methods have been explored to decrease the misuse and abuse of prescription opioids in the past decade. One such method is pre-operative "opioid counseling," which consists of educating patients on the effects and risks of opioid use, recommending appropriate opioid usage, and discussing alternative non-opioid pain treatment options. It has been shown that the counseling should be given as early as possible in the patient's surgical care and/or hospital stay, as a rushed education at discharge may be less effective. ${ }^{7}$ Orthopedic trauma patients who were pre-operatively counseled to cease opiate use by 6 weeks were found to be significantly more likely to do so than those without counseling. ${ }^{8}$ However, these findings have not been validated 
in Hand Surgery nor been shown to consistently affect opioid utilization patterns postoperatively. Moreover, it is important to understand that patients receiving prescription opioids are a heterogeneous group, and certain subsets of patients may benefit more from pre-operative counseling than others based on their prior opioid exposure. ${ }^{9}$

The purpose of this study was to assess the effect of pre-operative opioid counseling in hand surgery. The study was organized to analyze post-operative opioid use following CTR surgeries performed on patients who received pre-operative opioid counseling (the study arm) versus those who did not receive any formal counseling (the control arm). A hypothesis was made that patients receiving pre-operative opioid counseling would use less of their prescribed opioid and terminate use sooner compared to patients who did not receive any counseling.

\section{MATERIALS AND METHODS}

After Institutional Review Board approval, a prospective, randomized comparison of all consecutive CTR surgeries performed by the same hand surgery fellowship-trained board certified orthopedic surgeon, at the same surgical center, and using the same surgical technique was undertaken. Inclusion criteria included all cases of CTR surgery performed under local anesthesia only, on an outpatient basis. Exclusion criteria included any CTR surgery performed with sedation, or performed with any additional concomitant procedures, or if the patient had any history of pre-operative opioid consumption or an opioid allergy. Patients were randomized using a simple randomization scheme by birth year. Patients born with an odd birth year were assigned to the counseling (study) group, while those born with an even birth year were assigned to the no counseling (control) group. 
All data was retrieved through an email survey sent to patients at post-operative day three. Data collected included pain level by a 0-10 numerical scale and number of prescribed pills taken on each of the day of surgery and the first three post-operative days. Additional data collected included patient demographics, the presence of any adverse reactions to the prescribed opioids, and whether any other opioid or non-opioid painkillers were consumed. If a patient did not submit their response in a timely manner prior to their first post-operative visit, the survey was completed in the office.

All patients were prescribed 10 pills of Tylenol \#3 (325mg of Acetaminophen and 30mg of Codeine) post-operatively.

\section{Counseling}

Pre-operative opioid counseling consisted of reviewing the same one page informational form immediately prior to surgery on the day of surgery with the surgeon personally. The counseling form was derived from the recommendations defined by the Pennsylvania Orthopaedic Society (Fig. 1). The counseling consisted of explaining the significance and problems associated with the opioid epidemic. The counseling also consisted of making 5 formal pre-operative recommendations for the patient to consider, including: (1) Determining if the patient has any risk factors for opioid abuse. (2) To take non-opioid therapy prior to utilizing the prescribed opioids. (3) To understand the anticipated duration of typical opioid consumption surgery after CTR surgery. (4) To understand that the lowest dose opioid will be prescribed. And, (5) to declare if any opioid painkillers are also being prescribed by other providers.

\section{$\underline{\text { Surgical Technique }}$}

The same "mini-open" CTR surgical technique was utilized in all cases for both groups. The procedure was performed under local anesthesia alone, with 10-15 $\mathrm{cc}$ of $1 \%$ Lidocaine with 
epinephrine and $1 \mathrm{cc}$ of $8.4 \%$ Bicarbonate. A $2 \mathrm{~cm}$ longitudinal incision was placed at the base of the volar hand in line with the third webspace. The superficial palmar fascia was cut in line with the skin incision. The transverse carpal ligament was identified and released longitudinally until complete decompression of the median nerve was confirmed. The skin incisions were closed with three horizontal mattress 4-0 nylon sutures followed by application of soft sterile dressing that was recommended to be left in place and dry for two days.

\section{Statistical Analysis}

Hypothesis testing was performed using Fisher's exact test for categorical values and a student's t-test for continuous variables. Probability (p) values for the outcomes measures were generated with an analysis of variance (ANOVA). Statistical significance was defined as a p-value less than 0.05 .

\section{Source of Funding}

No external source of funding was used for this study.

\section{$\underline{\text { RESULTS }}$}

Based on pre-operative sample size estimate, a minimum of 20 patients were needed in each group to detect a pain score difference greater than 2, and a pill count difference of at least 1 pill. Consecutive patients were enrolled and randomized. Overall, 50 patients were approached, and 10 patients were excluded due to pre-operative opioid use or concomitant procedures. The study was concluded once 20 eligible patients were accrued in both groups. There were no Worker's Compensation patients in the series. The average ages were 61 and 62 years for the groups with and without counseling, respectively (Table 1). There were 11 females in the group with counseling (55\%) and 14 in the group without counseling (70\%). There was a 100\% 
response rate. There were no anesthetic complications or re-operations in either group. None of the patients reported taking any additional or different opioids after surgery other than the one prescribed. The difference in daily pain levels and daily opioid pill consumption is illustrated in Figures 2 and 3, respectively.

During the day of surgery and first post-operative day, patients in the group with counseling reported significantly fewer prescribed opioid pills consumed compared to patients in the group without counseling. In addition, patients in the group with counseling reported a significantly lower number of total pain pills consumed over the course of the study than the group without counseling. Twelve patients in the group with counseling consumed 0 opioid pills, while 4 patients in the group without counseling consumed 0 opioid pills over the course of the study. Patients reported no significant difference in pain level experience between the two groups on any day of the study. The reported opioid pill consumption and pain level experience for the entirety of the study are displayed in Tables 2 and 3, respectively.

Patients also reported non-opioid and non-prescription painkiller use (Fig. 4). These included Ibuprofen, Naproxen, and Acetaminophen. On the day of surgery, 12 patients in the group with counseling and 7 patients in the group without counseling consumed a nonprescription painkiller preferentially over their prescribed opioid. On the first postoperative day, 15 patients in the group with counseling and 10 patients in the group without counseling consumed a non-prescription painkiller. On the second postoperative day, 10 patients in the group with counseling and 8 patients in the group without counseling consumed a nonprescription painkiller. On the third postoperative day, 9 patients in the group with counseling and 9 patients in the group without counseling consumed a non-prescription painkiller. 
In addition, 0 of 20 patients in the group with counseling and 1 of 20 patients in the group without counseling reported adverse reactions to their prescribed pain killer. The single adverse reaction noted was minor and consisted of constipation. There were no cases of allergic reactions or major adverse reactions requiring hospitalization or re-operation in either group.

\section{$\underline{\text { DISCUSSION }}$}

Following upper extremity surgeries, including CTR, post-operative pain is typically managed with prescription opioids, often in excess of the patients' true needs. ${ }^{6,10}$ Specifically, $76 \%$ of patients undergoing CTR surgery have filled at least one prescription for opioid analgesics and $14 \%$ have filled a prescription beyond 90 days postoperatively. ${ }^{4}$ With a recent national focus on the increasing abuse of prescription opioids in the United States, the use and effectiveness of pre-operative opioid counseling has come under investigation. As this issue continues to garner attention from the medical community, more physicians have begun implementing opioid counseling prior to surgeries. ${ }^{6}$

Overall, opioid consumption was low in both groups, $1.40 \mathrm{v} 4.20$ opioid pills in the counseling and non-counseling groups, but was consistent with the findings by Chapman et al who identified an average opioid consumption of 4.3 pills after CTR surgery. ${ }^{11}$ In our analysis of pre-operative opioid counseling prior to CTR surgery, we found that patients with counseling consumed significantly less prescribed opioid pills than the patients with counseling on the day of surgery, on the first post-operative day, and in total over the course of the study (Table 2). Moreover, we found no significant difference in pain level between the two groups on any day of the study (Table 3), validating equivalent pain experiences and supporting the benefit of counseling on opioid consumption. In addition, more patients in the group with counseling used 
non-prescription painkillers than the group without counseling (Fig. 4). This, in conjunction with the similar pain levels in the two groups, also suggests that patients with counseling turned to non-prescription painkillers more readily to attain a manageable level of pain.

The study was designed only to investigate the effect of prescription opioid consumption and pain experience following CTR surgery in patients with and without pre-operative opioid counseling. Therefore, no outcome measures, patient satisfaction scores, or additional follow-up data were collected. The strength of the study was its prospective and randomized nature, a consecutive patient series, a $100 \%$ response rate, and minimization of biases. The study minimized potential biases by consisting of a single surgeon, use of the same single surgical technique, utilizing the same counseling form and script, and by the avoidance of sedation and the subsequent introduction of additional opioid / analgesic exposure.

Our study has some limitations. First, this study is based solely on the experience of a single surgeon at a single institution. Multi-center, prospective, randomized trials could build upon our data and provide further insight into the use of pre-operative opioid counseling. Furthermore, the study only evaluated Tylenol with codeine, and thus the findings may not be applicable to all opioids. In addition, the study might suffer from recall bias where patients misreport or underreport their actual pain experience or opioid pill consumption. Also, the study did not measure overall patient satisfaction with pain management, thus it is unclear at this time whether providing counseling alters patients' satisfaction with their operation.

Based on the study findings, we would recommend that surgeons consider routine preoperative counseling of their patients to help minimize opioid use. Also, based on our findings, we would recommend prescribing no more than 5-10 opioids post-operatively after CTR surgery. 


\section{Legend}

Figure 1 - Patient opioid counseling form.

Figure 2 - Postoperative pill consumption for counseling versus no counseling.

Figure 3 - Postoperative pain level for counseling versus no counseling.

Figure 4 - Postoperative consumption of non-prescription painkillers for counseling versus no counseling.

Table 1 - Demographic Characteristics

Table 2 - Postoperative pill consumption for counseling versus no counseling.

Table 3 - Postoperative pain level for counseling versus no counseling. 


\section{REFERENCES}

1. Kolodny A, Courtwright DT, Hwang CS, et al. The prescription opioid and heroin crisis: a public health approach to an epidemic of addiction. Annu Rev Public Health. 2015;36:559574.

2. Calcaterra S, Glanz J, Binswanger IA. National trends in pharmaceutical opioid related overdose deaths compared to other substance related overdose deaths: 1999-2009. Drug Alcohol Depend. 2013;131(3):263-270.

3. Manchikanti L, Helm S, Fellows B, et al. Opioid epidemic in the United States. Pain Physician. 2012;15(3 Suppl):ES9-ES38.

4. Johnson SP, Chung KC, Zhong L, et al. Risk of Prolonged Opioid Use Among Opioid-Naïve Patients Following Common Hand Surgery Procedures. J Hand Surg Am. 2016.

5. Wright EA, Katz JN, Abrams S, Solomon DH, Losina E. Trends in prescription of opioids from 2003-2009 in persons with knee osteoarthritis. Arthritis Care Res (Hoboken). 2014;66(10):1489-495.

6. Kim N, Matzon JL, Abboudi J, et al. A Prospective Evaluation of Opioid Utilization After Upper-Extremity Surgical Procedures: Identifying Consumption Patterns and Determining Prescribing Guidelines. J Bone Joint Surg Am. 2016;98(20):e89.

7. Costello M. Prescription Opioid Analgesics: Promoting Patient Safety with Better Patient Education. Am J Nurs. 2015;115(11):50-56.

8. Holman JE, Stoddard GJ, Horwitz DS, Higgins TF. The effect of preoperative counseling on duration of postoperative opiate use in orthopaedic trauma surgery: a surgeon-based comparative cohort study. J Orthop Trauma. 2014;28(9):502-506. 
9. Weiss RD, Griffin ML, Potter JS, et al. Who benefits from additional drug counseling among prescription opioid-dependent patients receiving buprenorphine-naloxone and standard medical management? Drug Alcohol Depend. 2014;140:118-122.

10. Rodgers J, Cunningham K, Fitzgerald K, Finnerty E. Opioid consumption following outpatient upper extremity surgery. J Hand Surg Am. 2012;37(4):645-650.

11. **B Blinded by JHS** 